\title{
Biophysics of Cryopreservation
}

\author{
Sharma Yashaswi ${ }^{1}$, Sharma Mona ${ }^{2 *}$ \\ ${ }^{1}$ MSc, Department of Reproductive Biology, AIIMS, New Delhi \\ $2^{*}$ Associate Professor, Department of Reproductive Biology, AIIMS, New Delhi \\ Email: dr.mona18sharma@gmail.com
}

Received 21 April 2021, Revised 30 May 2021, Accepted 6 June 2021

\begin{abstract}
A significant credit towards today's scientific and medical advancements goes to the technique of cryopreservation. Cryopreservation refers to the maintenance of cellular life at subzero temperatures for a definite period of time in a state of suspended cellular metabolism. The technique has become an indispensable step in most scientific research and medical applications like assisted reproduction, transplantations, and cell-based therapies where-in it allows the long-term preservation of biological specimens like gametes, embryos, viruses, cells and tissues. Although already an extensively used technique, a significant proportion of the cryopreserved samples still incur notable damage. Ultimately this leads to a decreased post-thaw viability and proliferation. Moreover, it is also possible that events during the freezing process, provoke more serious disturbances in the preserved material with regard to its identity and functionality. Hence, with the need to use the technique more judiciously, additional studies are needed for optimizing the current cryopreservation methods in use. For this, a thorough understanding of the normal physiological changes that the cryopreserved sample undergoes and the physics of cryopreservation seems plausible. The review thus aims to unravel the current knowledge on the complex physico-chemical processes and reactions that occur during the standard cryopreservation techniques.
\end{abstract}

Keywords: Cryopreservation; vitrification; freezing; thawing; biophysics.

\section{Introduction}

Cryopreservation aims to store cells indefinitely by arresting their metabolism and retarding the cellular reactions by means of low subzero temperatures. Such ultralow temperatures ensure that the cells are maintained below the glass transition temperature.

\section{The Underlying Biophysics of Cryopreservation}

\subsection{Water and its Transition to Ice}

Like in the biology of all living cells, water play a central role in cryobiology too. A cell consists of around 60 to $85 \%$ water both in free and bounded forms. The bound form refers to the water hydrated to complex mixtures of cells like proteins, lipids and ( $\mathrm{Tg}$ ) of pure water and no detectable biochemical activity is possible due to lack of sufficient thermal energy. Moreover, the progressive reduction and ultimately the absence of liquid water (once completely frozen) limit all metabolic processes [1,2].

Freezing is removal of water so that it transforms the liquid water into ice either when within the cell or after it flows out of the cell and freezes externally. However, it should be noted that freezing only affects free water in cells and not the bound form [3].

The protons and the oxygens of multiple water molecules are attracted electrostatically which form numerous weak hydrogen bonds. These bonds are highly dynamic and undergo frequent breaks and re- formations especially at its interface with other molecules and on the surfaces [3]. However, on cooling water below its freezing point, it resists breaking of hydrogen bonds. Rather molecules lock each other tightly to get organize into lattice like symmetry. This give rise to solid crystalline structures called ice which is less dense and occupy larger volume than liquid water.

\subsection{It all begins with nucleation}

Water in its pure form when cooled below its freezing point is undercooled or more commonly said to be supercooled until it is disturbed. However, once the local thermal properties allow hydrogen and oxygen molecules to come together to form sufficient $\mathrm{H}$-bonds, they initiate to form an 'ice embryo' [4]. This is called nucleation wherein molecules come close together and congregate in an arrangement which defines the crystal structure of the solid. The embryonic ice is thermodynamically capable to grow into a full-fledged ice as shown in Figure 1 [5].

Post nucleation, multiple water molecules complex with each other thereby forming networks that result in the exponential growth of the structure. Two types of nucleation exist depending upon the presence of a nucleation site [4]. The most common nucleation is the heterogeneous type wherein ice begins to form around a mere physical disturbance or an impurity like salt in the liquid or an irregularity in the container that act like a defined nucleation site.

Homogenous nucleation, on the other hand occurs only in pure water wherein ice forms without a predefined site of nucleation or a seed crystal [5]. Given to the rarity of pure water in biological samples, almost all nucleation is always heterogeneous. The types of nucleation are shown in Figure 2. 

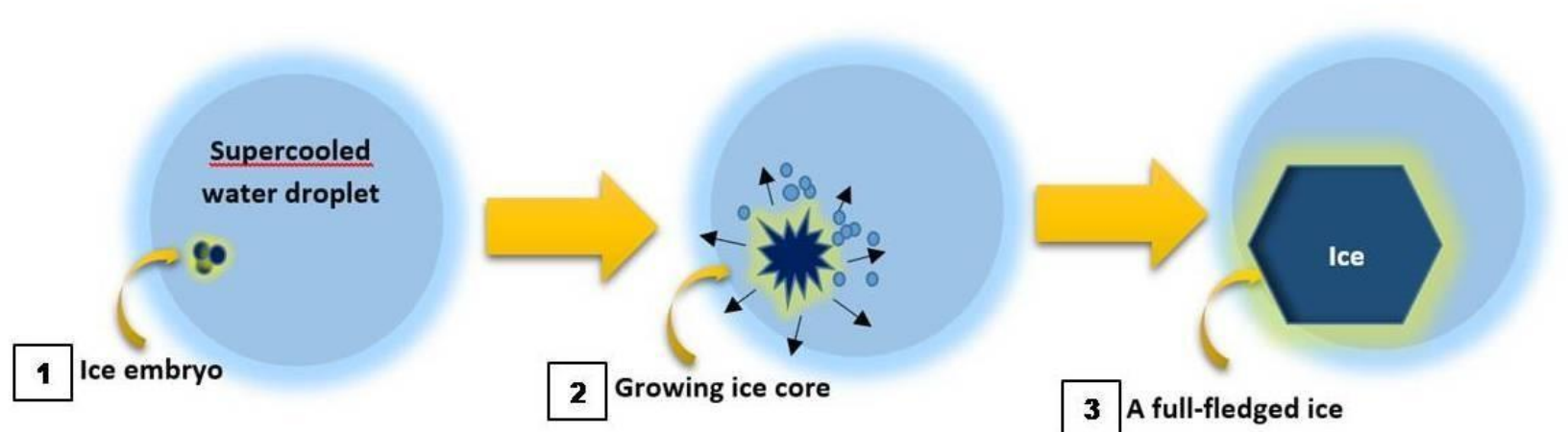

3 A full-fledged ice

Figure 1. The process of nucleation. 1. Formation of a nucleation site (an ice embryo). 2. The congregation of water molecule complexes on the embryo site to form an ice core. 3. The growth of a full fledged ice crystal wit regular symmetry.

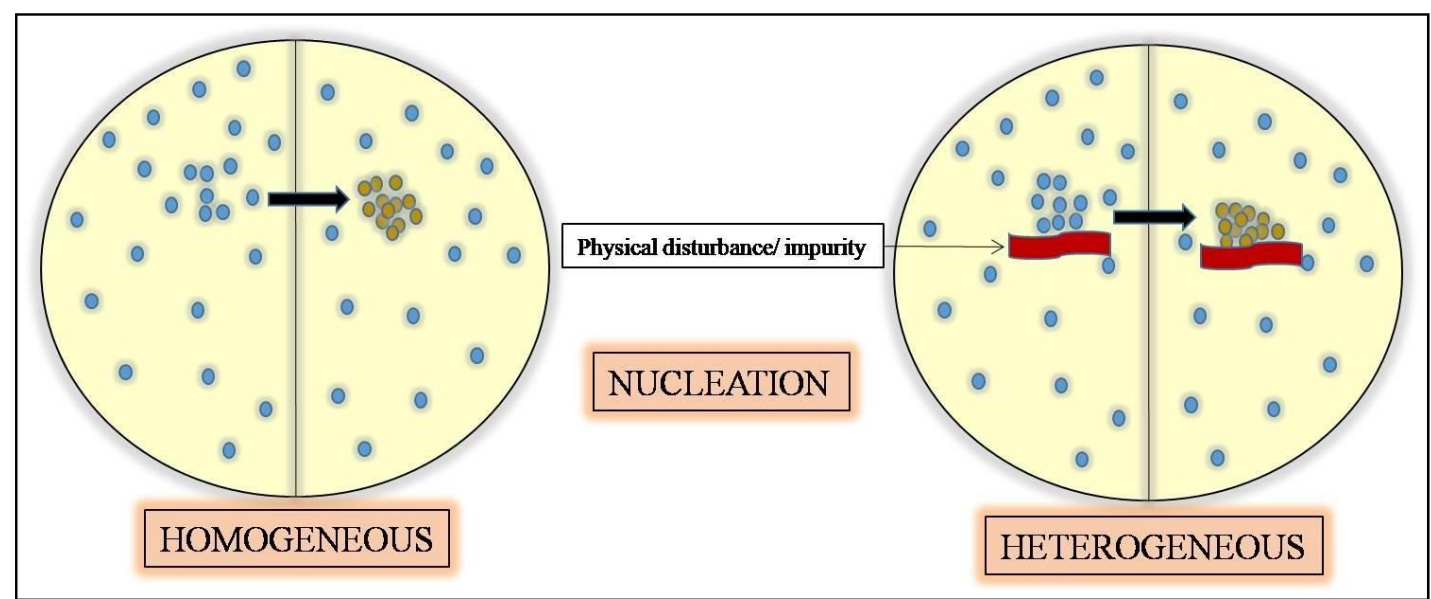

Figure 2. Types of nucleation: Homogenous nucleation occurs only in pure water wherein ice forms without a predefined site of nucleation or a seed crystal; heterogeneous nucleation wherein ice begins to form around a physical disturbance or an impurity or an irregularity in the container that act like a defined nucleation site.

\subsection{Osmosis follows Ice formation}

Cryobiology not only concern with the state of water in the cells but also involves its movement and the factors that govern such movement of water into and out of the cells during the freezing process. The osmotic relations of the cell abides by the principle that in the absence of metabolic forces, the concentration of water and solutes in the cell is adjusted such that both are in a chemical potential equilibrium with the concentrations outside the cell [3].

Hence, the two kinetic processes occurring during the cooling of cells that is growth of ice and the loss of water from the cell, happens at a characteristic rate [3]. This is highly influenced by the cooling rate imposed on the system $[6,7]$. If cooling is provided at a slow constant rate (such as during conventional freezing), it allows the cell to remain close to the osmotic equilibrium and the rate of water loss from the cell will be in accordance to the rate of water loss from extracellular solution as it changes to ice. However, if a rapid cooling rate is applied, the two rates will vary significantly. The intracellular water freezes to ice at a faster rate due to the absence of a permeability barrier which the water inside of the cells need to surpass. This leads to a continuous increase in the osmolality gradient across the plasma membrane [6-8].

\section{Biophysics of Conventional Slow Freezing and Rapid Freezing}

Freezing usually occurs only when it is energetically and physically favorable that is when ice templates are available for it to do so [4]. With the drop in temperature associated with the cryopreservation process, ice is preferentially formed extracellularly (except when cells undergo rapid freezing). As the extracellular liquid water transitions to a solid state, the solute concentration of the system increases. This creates a deficit between the partially frozen outside of the cell and the unfrozen inside. This in turn induces the efflux from the inside of the cells to establish the osmotic equilibrium [3]. In case a controlled reduction of temperature is maintained, a sufficient osmotic pressure persists that prevents the formation of ice crystals within the cell. However, the cells continually shrink during the process due to the efflux of water [9]. During rapid freezing, there is less time for water to move in the extracellular compartment and gets supercooled very fact leading to intracellular ice formation. Therefore, an optimum cooling rate is essential. Rapid freezing leads to cell death due to intracellular ice formation. Slow freezing leads to cell exposure to hypertonic environment thereby causing water efflux and cellular dehydration. The mechanism is explained in Figure 3. 


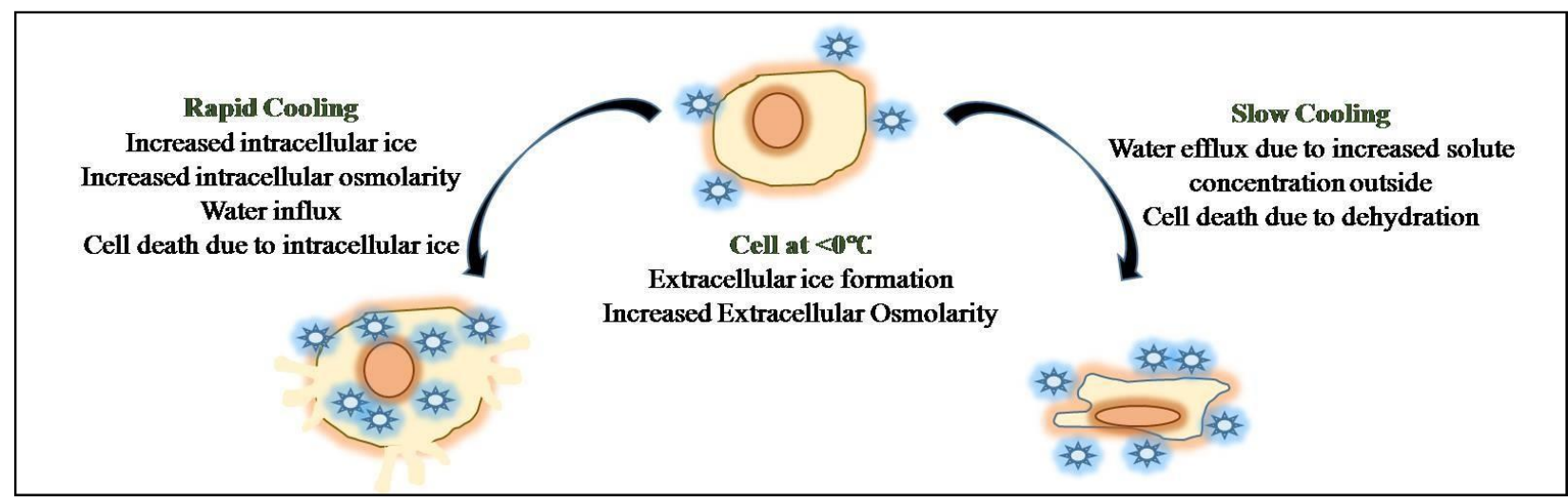

Figure 3. The consequences of different cooling rates imposed on cells undergoing cryopreservation.

\section{Biophysics of Vitrification}

Viscous liquids allow rapid cooling far below their melting temperature and undergo solidification by avoiding crystallization [10-12]. This property is utilized in the process of vitrification. The supercooled substance with the physical properties of a liquid subsequently acquires solid properties once it reaches below a particular temperature called the glass transition temperature (Tg) [10]. At this point, the molecules of the substance remain in a disordered pattern as in liquids but are locked in place and the consequent "solid-liquid" is called as the glass [11]. Since there is no crystallization event, vitrification outruns the processes of ice nucleation and growth and thus their potential adverse effects [12].

With continuous exposure to low temperatures, the energy of molecules progressively dissipates with time such that they are unable to move amongst each other. Hence the molecular motion ceases and at $\mathrm{Tg}$, they are physically confined (although still disordered) and vibrate at their positions mimicking the confinement within crystals. With subsequent temperature fall, the viscosity of the system significantly increases (as cell solutes become concentrated) [11]. The increased viscosity prevents the water molecules coming together to initiate ice formation [10]. With this, the values of heat capacity, thermal expansivity, and compressibility resemble to those of a crystal and hence the substance results into an amorphous solid [11].

\section{Biophysics of Thawing}

The rewarming process also encompasses change in ice stability, size and structure. However, the exact magnitude and type of change depends on the type of specimen, the cooling rate and the cryoprotective additives used in the process. More significantly, the events of warming vary in terms of type of cryopreservation method employed. As the ice melts, the solute concentration in the residual phase is decreased. For cells that underwent slow freezing and thus the dehydration process, a progressive rehydration step occurs during warming [3]. This is opposed to cells frozen at high cooling rates since they are not in osmotic equilibrium during the freeze. They thus undergo further dehydration in response to the decreased extracellular solute concentration during thaw. But as more ice melts, the extracellular solution is diluted, causing the reversal of the water flux and ultimately cell rehydration [3].

A significant event during rewarming that might result in increased cell lethality is recrystallization in cells with intracellular ice. It should be noted that small ice crystals have a higher internal pressure than the larger ones due to increased interfacial curvature and ice-solution interfacial tension. Since this high pressure influences chemical potential, large ice crystals may grow while small ice crystals are melting at a given temperature causing further damage to cells [6].

\section{Devitrification}

Successful devitrification is a challenge. The glassy solid is extremely fragile and can revert back either to a liquid or devitrify to form ice [13]. The ice formation may be a consequence of the changes in molecular mobility of rewarmed water molecules which under sufficient energy conditions, can relax and rearrange themselves to form ice. Further the glass may simply crack or fracture causing significant damage to organelles $[14,15]$.

Hence in practice, most vitrification protocols employ a rapid rate of warming such that ice nucleation is inhibited while passing through the temperature of glass transition $[16,17]$. However, utmost concern must be given since too rapid rewarming increases the possibility of stress cracks and fractures. Ideally employing a two phase procedure, in which a short slow rewarming phase lasting a mere few seconds followed by a rapid warming phase at a high temperature looks promising for successful devitrification [18].

\section{Mechanism of Cryoinjuries}

There may be multiple effects of ice formation such as cellular dehydration, mechanical stress by extracelluar ice, intracellular crystallization, thermal shock etc. The extent to which the cryopreservation technique will succeed depends on the immediate post-thaw survival rate and the effect of the freeze thaw process on cryopreserved cells. However, the other subtle effects on DNA, mRNA and protein function may not be observed immediately postthaw. DNA quality and gene expression are crucial factors for cell development. Cells undergoing cryopreservation are exposed to extreme physical and chemical conditions that may alter cell integrity at molecular level.

There have been studies on molecular effects of cryopreservation targeting DNA damage and altered gene expression. Few study group showed no adverse effects of cryopreservation in the porcine and humans [19] whereas other groups showed negative effects in the ovine and human species [20,21]. The extensive work done in different cell types such as sperm, oocytes, erythrocytes and leukocytes showed that cell quality declines after the freeze-thaw process due to DNA damage and fall in mRNA levels due to addition of cryoprotectants, cryo injury, intracellular ice crystallization [20, 22,23]. 


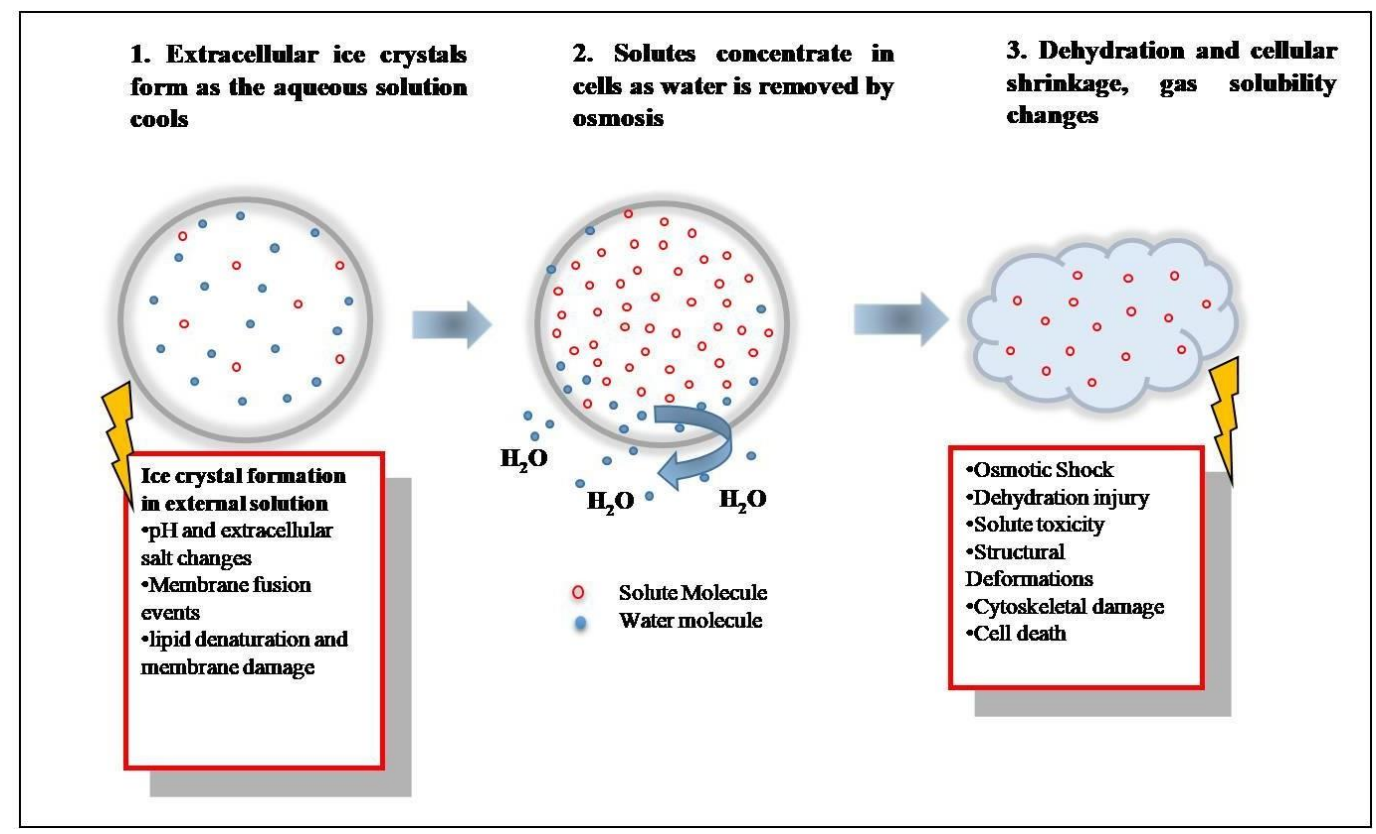

Figure 4. The process of slow freezing of cells and its cell injury mechanisms.

\subsection{Biophysics of Slow-Freezing Injury}

During slow cooling, the cell is able to sustain the osmotic equilibrium by balancing the rate of water loss from cells to the rate of extracellular ice formation [3] Although this prevents the problem of intracellular ice formation, it encompasses considerable cell dehydration. Two important mechanism of cell injury is noted during slow freezing: Solute toxicity and physical changes due to cell dehydration $[7,8,24-27]$. The two major consequences of dehydration are a) increased extracellular solute concentration denaturing membrane proteins b) salt crystallization may also bring $\mathrm{pH}$ changes that may again denature proteins.

Solute toxicity arises due to the increased solute concentration in cells. During slow cooling process, solutes increase in concentration both extracellularly and intracellularly due to the formation of external ice and the resulting efflux of water from the cells [28]. This can lead to alterations in the chemical equilibrium of the cell through varied biophysical and biochemical changes leading to cell death. For instance, highly concentrated salt can have a lyotropic effect on the cell membrane [19]. The mechanism of cell injury in slow freezing is shown in Figure 4.

On the other hand, cell shrinkage is capable of causing structural deformations among other defects. The dehydration of the plasma membrane due to water loss may cause membrane fusion events resulting in parts of membrane being internalized as vesicles and consequently decreasing the surface area of the cell adversely affecting its housekeeping functions [27]. Besides this, there are many theories as to how cell dehydration causes damage to the cells. The minimal volume hypothesis states that once the cell reaches its minimum volume after water efflux, it fails to maintain the osmotic equilibrium and thus the gradient difference can only be minimized by salt movement from extracellular solution into the cytoplasm. On reversal to isotonic condition during thawing, the cytoplasm with higher concentration of solute would draw water in to reach equilibrium. If this expansion surpassed the yield strength of the membrane, the cell undergoes lyses [29]. A variant of the same theory suggests that once the minimum volume is reached, the induced hydrostatic pressure gradient causes a mechanical stress on the cell resulting in injury [26].

Nevertheless, formation of large ice crystals even extracellular can have grave consequences. The expansion can cause pressure and has a shearing effect on membranes resulting in appreciable damage [9].

\subsection{Biophysics of Rapid Freezing Injuries}

Intracellular ice formation is the most well-known cell injury inflicted by higher rates of cooling [30]. As described earlier, during a rapid freeze protocol, a cell is unable to maintain equilibrium with the external environment since extracellular ice forms too quickly to balance by exosmosis. As a result, the cytoplasm is increasingly super-cooled which in turn increases the chance of allowing nucleation and thus intracellular ice formation. [30,31] The nucleation has been shown to result from interactions of extracellular ice with the plasma membrane. A set of hypotheses explain that the external ice with an appropriate tip radius grows through the aqueous or proteinaceous pores of plasma membrane to seed the cytoplasm for intracellular ice formation [31-33]. This results in cell injury due to the enlargement of the membrane pore on re-crystallization during warming. This is well explained under the proteinpore theory [32]. Alternatively, it could also be that membrane damage precedes intracellular ice formation (the membrane failure hypothesis) as shown through the work on unfertilized eggs of sea urchin [34]. The membrane may be damaged either by development of electrical transients created by charge separation at the interface of the growing ice and the aqueous solution or through the virtue of a critical osmotic pressure gradient across the membrane $[35,36]$. It has also been hypothesized that the plasma membrane could act as an effective nucleator for internal ice when acted on by extracellular ice. This is known as the surface- catalyzed nucleation theory [37].

It is widely believed that formation of intracellular ice is lethal to cells mostly due to the mechanical damage they impose via a surface-area-to-volume redistribution of the ice crystal $[7,13,38]$. This has been believed to damage 
internal organelles of the cell. As a matter of fact, the hazardous effect of ice crystal formation is more prominently evident during the warming process. A slow thawing protocol results in the recrystallization of the ice, increasing its size. Recrystallization, however, is not the only means by which intracellular ice can be lethal. Intracellular ice formation also include non-mechanical modes of destruction such as through solution effects, thermal shock, osmotic injury, protein denaturation, and gas bubble formation [39-44]. However, the degree of lethality and damage incurred is strictly influenced by the amount, size, location and mechanism of formation of such ice crystals as described in Figure 5 [40,45,46].

\section{The Role of Cryoprotectants}

A range of chemical substances with high solubility that work to protect biological cells from cold shock have been employed to substantially increase the survival following freezing and thawing [47]. These substances called cryoprotective agents or cryoprotectants are either permeating or non- permeating [1] type depending upon their mode of action.

\subsection{Mode of action of Permeating Cryoprotectants}

Permeable cryoprotectants diffuse through the plasma membrane and equilibrate in the cytoplasm. They have a low molecular weight and are non-ionic with low cellular toxicity $[9,48]$. They are most commonly employed to mitigate slow-cooling injuries and primarily work by decreasing the freezing point [48]. They lower the amount of ice that forms at a given temperature by forming $\mathrm{H}$-bonds with water molecules [3]. This in turn lowers the salt concentration found normally in physiological solutions for a given temperature (below the freezing point, when ice formation causes the concentration of these salts). Besides, they also act as secondary solvent for the salt present [3]. Hence by depressing the temperature at which the cell is exposed to increased extracellular solute concentration, the magnitude of injury, and the kinetics at which damage accumulates, is reduced. Further, it has also been seen that permeable cryoprotectants enters the cell and increases its viscosity. This helps preventing the cell reaching the minimum lethal cell volume due to dehydration $[48,49]$.

\subsection{Mode of action of Non-permeating cryoprotectants}

Non-permeating cryoprotectants are bulky long-chain polymers that remain extracellular and are used mostly for rapid freeze processes [50]. They have large osmotic coefficients and use this property to dehydrate cells before freezing which then requires lesser amount of water loss from the cell to maintain osmotic equilibrium during cryopreservation [3]. As a result the cytoplasm do not supercool to the extent to initiate the formation of intracellular ice at the given cooling rate [3,9]. Figure 6 explains modes of action of permeating and non-permeating cryoprotectants.

\subsection{Cryoprotectants to achieve Vitrification}

Cryoprotective vitrification strategy involves the use of cryoprotectants to significantly increase the cell viscosity such that when a cell is exposed to cryogenic temperatures the nucleation event for ice formation is completely inhibited [51]. This can be achieved by the addition of cryoprotective additives at very high concentrations [49]. However, the toxicity of the cryoprotectant at the intended concentration must be taken into account [52]. In the recent years, sugars have found a utility in vitrification regimes as effective cryoprotectants especially for mammalian cells. They offer several advantages in regard to substantially increasing the cell viscosity and also protecting cells from shrinkage during the freezing process [53]. Sugars like sucrose, raffinose, trehalose etc have high molecular weights and are capable of forming hydrogen bonds with water. At high concentrations of monosaccharides and disaccharides, sugars render protection by formation of a stable glassy matrix and also by binding to sites previously stabilized by water [54- 59].

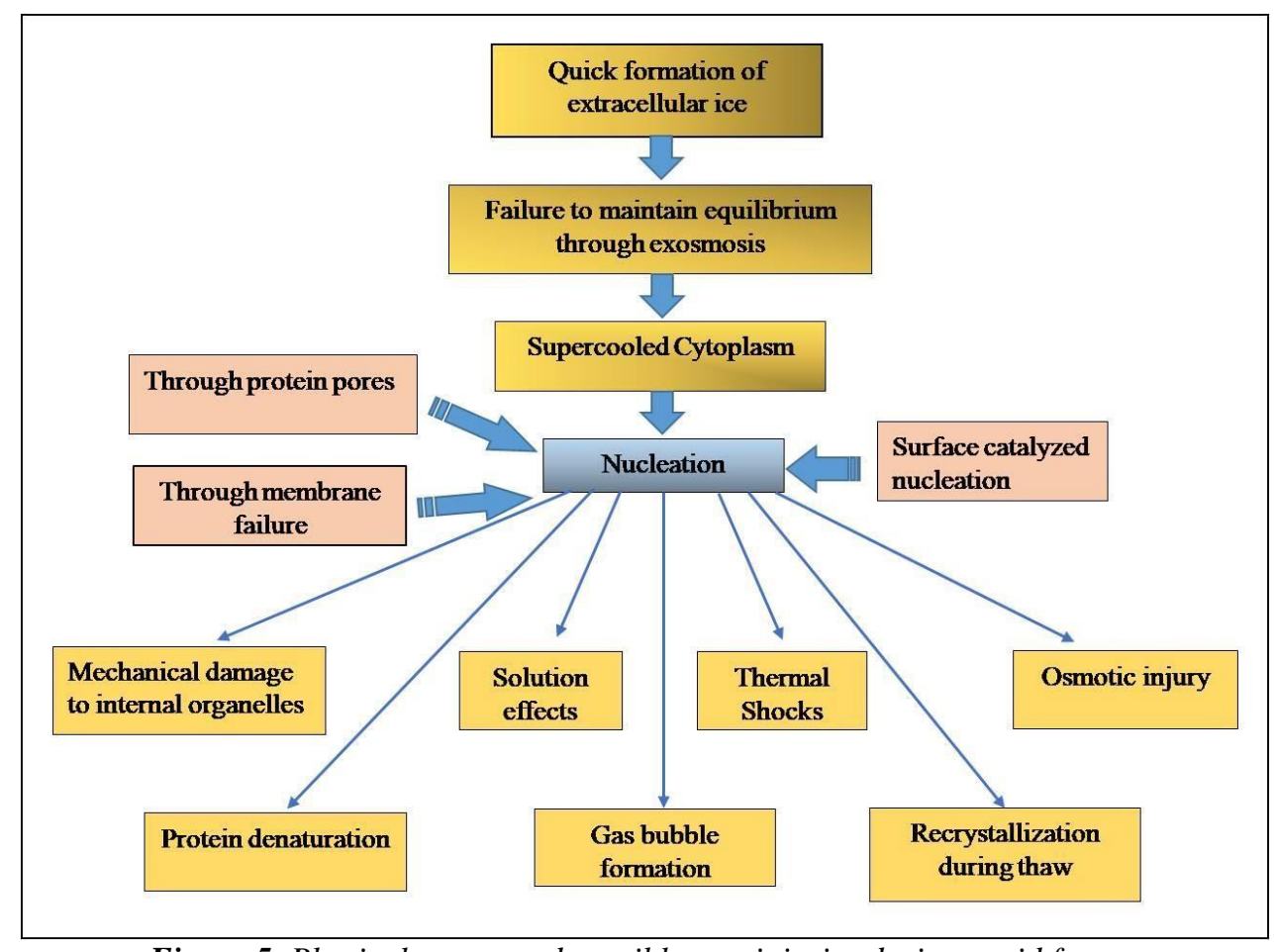

Figure 5. Physical events and possible cryoinjuries during rapid freeze. 


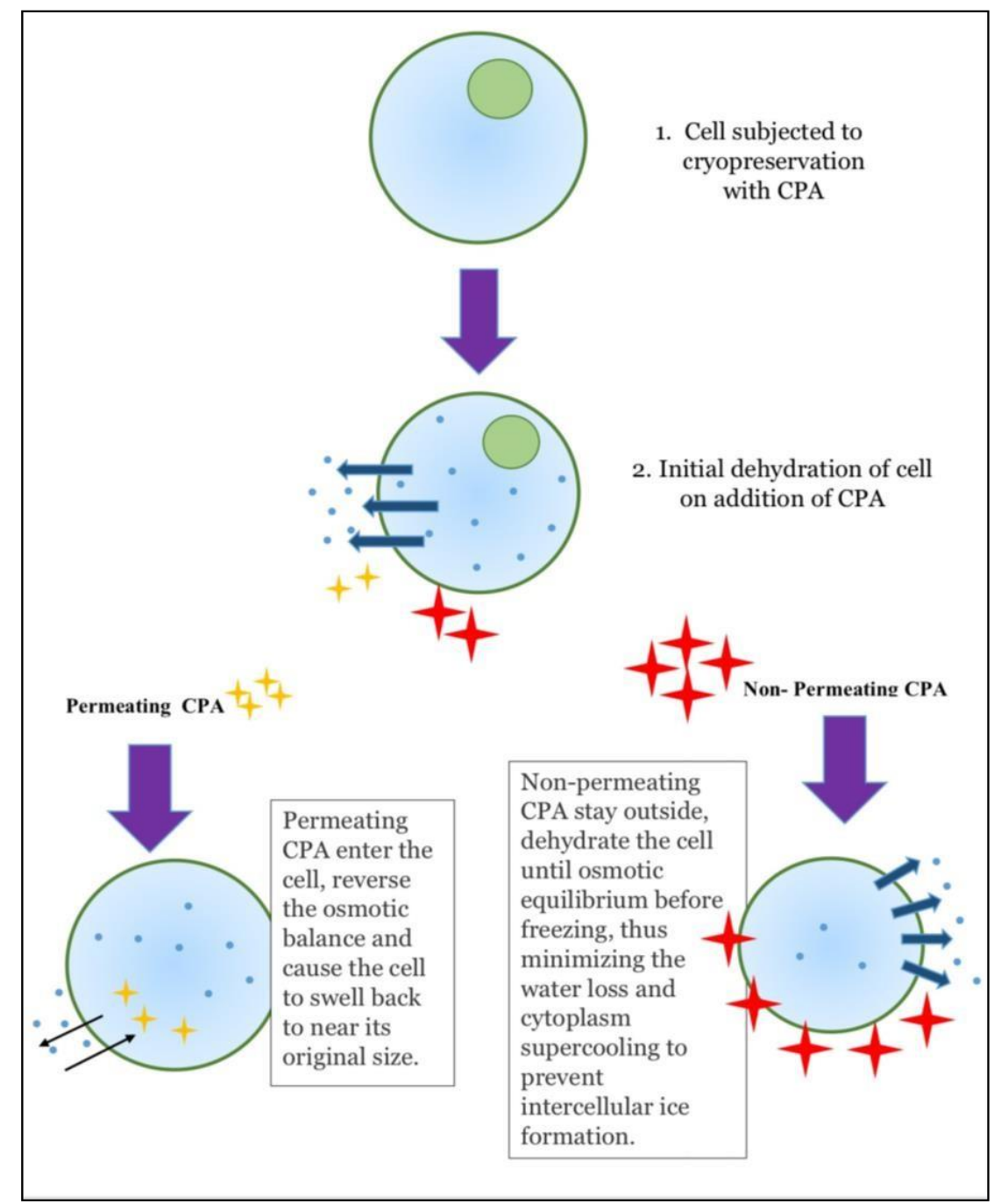

Figure 6. Mode of action of permeating and non-permeating cryoprotectants.

\section{Applications of Biophysics Understanding \\ 9.1 Selection of Cooling Rates}

The rate of cooling imposed during the cryopreservation process greatly determines the cell survival outcome [6062]. The effect can be explained by an underlying principle described as the inverted "U" of cryobiology (Figure 7). This is based on observations whereby graph plots of cell survival vs. cooling rate imposed on a system take the form of an inverted "U" [3]. This is because, only few cells survive when the cooling rate is very slow and even fewer survive when the rate is high. Cells remain most viable when the cooling rate is optimal which is specific for a cell type. Two opposing damaging factors account for the two arms of the inverted " $U$ ": the formation of intracellular ice crystals and dehydration injury. Formation of intracellular crystals is more likely and damaging when the cooling rate is high while dehydration damages are higher at low cooling rates [63].

The injury mechanisms that occur at both slow cooling rates and rapid cooling rates are shown. The maximal cell survival is obtained at the optimal cooling rate. When gradual cooling rates are imposed on cells, as discussed earlier, extracellular ice forms raising the solute concentration on the outside. This creates a differential water gradient and requires water from the interior of the cells to undergo exosmosis to relieve the gradient. This is the basis to the advantage of a slow controlled cooling. Since water is effluxed, the amount of water is reduced within the cell and the chance of more lethal intracellular ice crystal formation is hence minimized. Said differently, the formation of extracellular ice is advantageous since it inhibits the more lethal intracellular ice crystal formation. However, if the cooling rate is too slow, the solute concentration rises sharply causing colligative damage to the cells. Hence, an optimal cooling rate is to be chosen for best survival rates such that only a minimal water remains inside the cells to inhibit ice formation while just the right amount of it is to be removed to prevent colligative solution effects [3].

The real challenge however remains to select the optimal cooling rate since it varies with change in specimen cell type. For example, the optimum cooling rate for mouse marrow stem cells via a study may be concluded at $1^{\circ} \mathrm{C} / \mathrm{min}$ for but may be over $1000^{\circ} \mathrm{C} / \mathrm{min}$ for human red blood cells (RBCs) [3]. Further, the cell survival at a particular cooling rate is also largely determined by the warming and thawing procedure applied. In addition to that, the type of cryoprotectant used and the concentration it is used at further may exert a significant effect on the results.

\subsection{Controlling Ice Nucleation}

The most significant event in the course of conventional cryopreservation is the nucleation of ice and it has important consequences [4]. Ice nucleation is believed to affect the cell structural, osmotic and colligative properties as well as is capable of causing ruptures and mechanical injuries through same means [5]. 


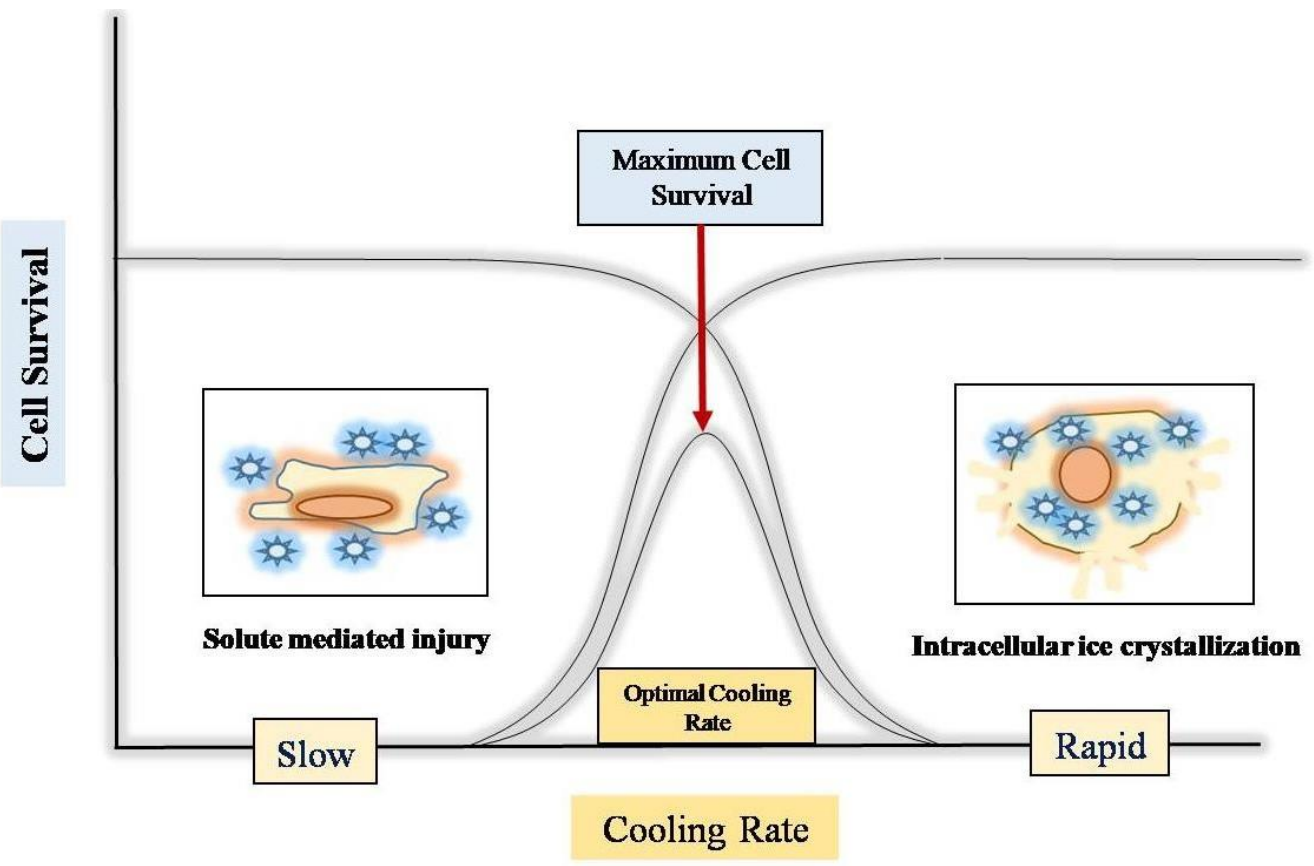

Figure 7. Cell Survival vs. Cooling Rate Curve.

"Seeding" refers to one such method wherein an external preformed ice crystal is deliberately introduced as a nucleator into the system at a particular temperature $[7,64,65]$. A cold spot is generated generally outside of the container to minimize contamination. Seeding works by preventing the supercooling of the specimen and spontaneous nucleation. If such initial transition of liquid to ice is site and time controlled, the subsequent events leading to damage is also surpassed [5]. Ice nucleators can also be formed with the use of 'chemical nucleants', added in the sample medium or by electro-freezing which utilizes a high voltage of electricity to induce crystal formation [6669]. Alternatively, some mechanical methods exists, which involve disturbing the solution by shaking or tapping [70]. Even the use of ultrasound waves for nucleation is feasible however, the standardization is difficult [71]. Other methods include using cold shocks or pressure shifts which involves exposing the specimen to rapid changes in temperatures and pressure respectively $[72,73]$.

\subsection{Selection of Cryoprotectants}

Today almost all cryopreservative regimes employ the use of some form of cryoprotective agents during the freezing process [51,52]. With the understanding of the physics of the cryoprotectants and their mode of action, a range of chemicals with one or more cryoprotective action are available. However, on understanding their working mechanism it was also clear that cryoprotectant under use must be non-toxic to the specimen being preserved [52]. This is especially true for permeating cryoprotectants. Nonetheless, non-permeating cryoprotectants also engender the risk of causing osmotic injury [51]. Cryoconservationists have thus been increasingly exploiting and amalgamating cryoprotectant modalities.

Considering the dynamics of freezing, any cryoprotective agent rendering colligative cryoprotection will be an effective cryoprotectant. If so, it might be able to control the cooling rate and optimize the degree of cell dehydration to avoid both ice damage (caused by insufficient water loss) and colligative damage (caused by excessive water loss) [3].
It has also been shown that the use of a mixture of different cryoprotective additives is advantageous since this might help in nullifying the toxic properties of individual cryoprotectants as well as render the system with synergistic cryoprotective action [74]. The use of a mixture of permeating and non- permeating cryoprotectant is already common for many cryopreservation protocols [1].

\subsection{Selection of Thawing and Rewarming Rates}

As discussed earlier, the temperature and mode of thaw have as important consequences as the process of freezing employed on specimen survival and proliferation. [75,76] The method chosen however depends on the method of freezing recruited, the cooling rate imposed and the type of cryoprotectants used (Figure 8). Taking into account the biophysical relations of the cells, in general, cells frozen at supra- optimal rates give higher survival when warmed rapidly [16,17]. This is because, a slow warming may cause recrystallization of ice and/or non-uniform expansions and contractions leading to thermal, colligative and osmotic stress. On the other hand, the cells which are cooled at suboptimal rates do not follow the trend (either work oppositely or are unaffected by the warming rate) [18].

\section{Increased Interest towards Vitrification}

Understanding the basic biology of cryopreservation is an essential step towards achieving successful survival of cells post-thaw [77]. Since cryopreservation by vitrification completely omits ice formation and requires minimal equipment it stands as a promising alternative to conventional methods of freezing [10,11]. From the standpoint of biophysics too, vitrification is highly valuable in that it omits the drastic molecular reorganization that happens in most other freezing strategies and preserves the natural disorder of water molecules and dissolved solutes in cells [11]. This can be highly beneficial since it will allow preserving cells closer to their natural state. Hence, vitrification has gained a lot of attention in the recent years. The key to a successful vitrification regime involves exposing cells to ultralow temperatures with a very high cooling rate, and ensuring arrangements that critically 
increase the cell viscosity to the point where ice formation is prevented and the water present turns into an amorphous glassy-solid [10] (Figure 8). This has been shown to be possible by either the use of cryoprotectants at very high concentrations or by the removal of water by techniques like evaporative desiccation and osmotic dehydration prior freezing; both with its own limitations $[9,10]$.

As far as achieving highest cooling rates are concerned, studies have shown that plunging samples directly from air into a cryogenic liquid such as nitrogen, propane or ethane by virtue gives the highest cooling rates (by a factor of 20100 over previous best practice) [78].

\section{Conclusion}

A robust protocol development for successful cryopreservation involves understanding the basic principles of the cryopreservation theory. Consequently, scientists can optimize their storage methods leading to enhanced long term preservation of varied specimen collections in a stable cryogenic state.

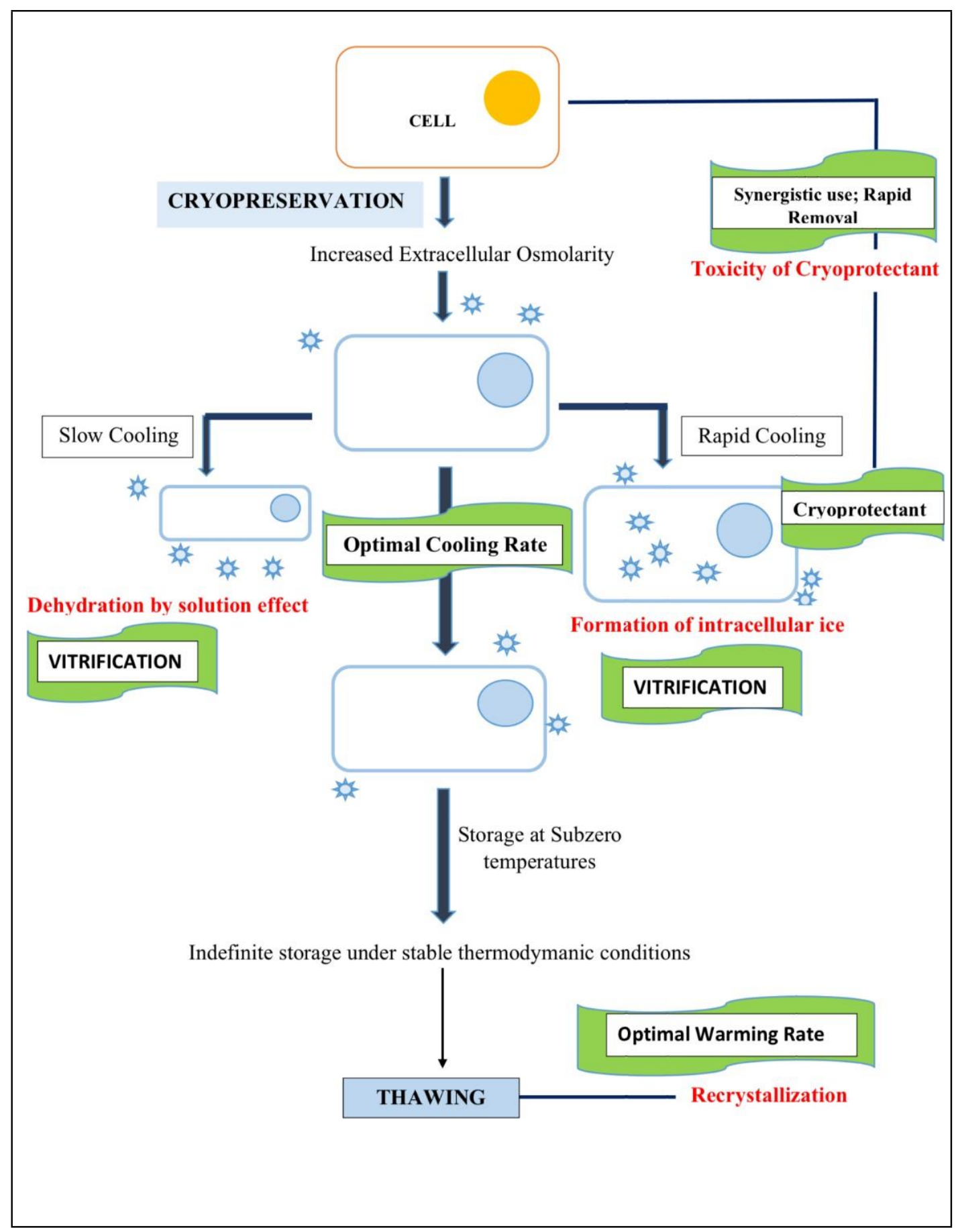

Figure 8. Physical events, possible cryoinjuries (in red) and solution to injuries (green) during cryopreservation processes. 


\section{References:}

[1] Semen Freezing Past Present and Future. $4^{\text {th }}$ Nexus Ebulletin.Indian Fertility Society \& Origio India Initiative. 2017.

[2] D. E. Pegg. Principles of cryopreservation. Methods Mol Biol, 368: 39-57, 2007.

[3] P. Mazur. Principles of cryobiology. in Fuller, B.J., Lane, N.L., Benson, E.E. (Eds.), Life in the frozen state. CRC Press LLC, Boca Raton, FL, pp 3-65, 2004.

[4] The Physics of Ice: It All Begins with Nucleation. Alex Esmon 12.18.2014. Thermofisher Scientific.

[5] G. J. Morris, E. Acton. Controlled ice nucleation in cryopreservation - A review. Cryobiology, vol. 66, No. 2, pp.85-92, 2013.

[6] P. Mazur. Freezing of living cells: Mechanisms and implications, Am. J. Physiol. 247, C125-C142, 1984.

[7] P. Mazur, S. Leibo, E. Chu. A two-factor hypothesis of freezing injury. Evidence from hamster tissue-culture cells. Exp. Cell Res, vol. 71, No.2, pp. 345-355, 1972.

[8] J. E. Lovelock. The haemolysis of human red blood cells by freezing and thawing. Biochim. Biophys. Acta. Vol.10, pp. 414-426, 1953.

[9] D.E. Pegg. Principles of cryopreservation. Methods Mol Biol. Vol. 1257, No. 3, pp.19, 2015.

[10] Principles of Cryopreservation by Vitrification Authors: Gregory M. Fahy Brian Wowk Series: Methods In Molecular Biology > Book: Cryopreservation and Freeze-Drying Protocols. 2015. Springer Protocols. Springer-Verlag New York. Humana Press. Willem F. Wolkers, Harriëtte Oldenhof (eds.).

[11] B. Wowk. Thermodynamic aspects of vitrification. Cryobiology. Vol. 60, No.1, pp. 11-22, 2010.

[12] N. Chao, I. Chiu Liao. Cryopreservation of finfish and shellfish gametes and embryos, Editor(s): Cheng-Sheng Lee, Edward M. Donaldson, Reproductive Biotechnology in Finfish Aquaculture, Elsevier, pp.161189, 2001.

[13] G. M. Fahy. Biological Effects of Vitrification and Devitrification. In: D.E. Pegg, A.M. Karow (eds) The Biophysics of Organ Cryopreservation. NATO ASI Series (Series A: Life Sciences), Springer, Boston, MA, vol. 147, 1987.

[14] S. Seki and P. Mazur. Effect of warming rate on the survival of vitrified mouse oocytes and on the recrystallization of intracellular ice. Biology of reproduction. Vol. 79, No. 4, pp. 727-737, 2008.

[15] W. F. Rall. Factors affecting the survival of mouse embryos cryopreserved by vitrification. Cryobiology. Vol. 24, No. 5, pp.387-402, 1987.

[16] A. S. Teixeira, M. E. González-Benito, A. D. MolinaGarcía. Measurement of cooling and warming rates in vitrification-based plant cryopreservation protocols. Biotechnol Prog. Vo. 30, No. 5, pp. 1177-84, 2014.

[17] S. Seki and P. Mazur. The dominance of warming rate over cooling rate in the survival of mouse oocytes subjected to a vitrification procedure. Cryobiology. Vol. 59, No. (1), 75-82, 2009.

[18] Reed, M. Barbara. Plant Cryopreservation: A Practical Guide. Springer Science \& Business Media, SpringerVerlag New York Inc, 2008.

[19] S. Hamamah, D. Royere, J.C. Nicolle, M. Paquignon, J. Lansac. Effects of freezing- thawing on the spermatozoon nucleus: a comparative chromatin cytophotometric study in the porcine and human species. Reprod. Nutr. Dev. Vol.30, pp. 59-64, 1990.

[20] S. Succu, D. Bebbere, L. Bogliolo, et al. Vitrification of in vitro matured ovine oocytes affects in vitro preimplantation development and mRNA abundance. Mol. Reprod. Dev. Vol.75, pp. 538-546, 2008.

[21] M. Tachataki, R.M.L Winston, D.M Taylor. Quantitative RT-PCR reveals tuberous sclerosis gene, TSC2, mRNA degradation following cryopreservation in the human preimplantation embryo. Mol. Hum. Reprod. Vol. 9, pp. 593-601, 2003.

[22] T.C Fisher, S. Groner, U. Zentgraf, V. Hemleben. Evidence for nucleosomal phasing and a novel protein specifically binding to cucumber satellite DNA. Z. Naturforsch. Vol. 49, pp.79-86, 1994.

[23] S.I Peris, A. Morrier, M. Dufour, J.L Bailey. Cryopreservation of ram semen facilitates sperm DNA damage: relationship between sperm andrological parameters and the sperm chromatin structure assay. J. Androl. Vol. 25, pp. 224-233, 2004.

[24] J. E. Lovelock. The mechanism of the protective action of glycerol against haemolysis by freezing and thawing, Biochim. Biophys. Acta. Vol. 11, pp. 28-36, 1953.

[25] H. T. Meryman. The exceeding of a minimum tolerable cell volume in hypertonic suspension as a cause of freezing injury, in The Frozen Cell, Wolstenholme, G.E. and O'Connor, M., Eds., Churchill, London, pp. 51-64, 1970.

[26] H. T. Meryman. Freezing injury and its prevention in living cells, Annu. Rev. Biophys. Vol. 3, pp. 341-363, 1974.

[27] P.L. Steponkus and S.C. Wiest. Plasma membrane alterations following cold acclimation and freezing, in Plant Cold Hardiness and Freeze Stress-Mechanisms and Crop Implications, Li, P.H. and Sakai, A., Eds., Academic Press, New York, pp. 75-91, 1978.

[28] P. Mazur. Kinetics of water loss from cells at subzero temperatures and the likelihood of intracellular freezing, J. Gen. Physiol. Vol. 47, pp. 347-369, 1963.

[29] A.M.M. Zade-Oppen. Posthypertonic hemolysis in sodium chloride systems, Acta Physiol. Scand. Vol. 73, pp. 341-364, 1968.

[30] J. A. Elliott. Intracellular ice formation: the enigmatic role of cell-cell junctions. Biophys J. Vol. 105, No. 9, pp. 1935-1936, 2013.

[31] P. Mazur. The role of cell membranes in the freezing of yeast and other single cells, Ann. N.Y. Acad. Sci. Vol. 125, pp. 658-676, 1965.

[32] J.P. Acker and L.E. McGann. Membrane damage 
occurs during the formation of intracellular ice, CryoLetters. Vol. 22, pp. 241-254, 2001.

[33] W.K. Berger and B. Uhrik. Freeze-induced shrinkage of individual cells and cell-to-cell propagation of intracellular ice in cell chains from salivary glands, Experientia. Vol. 52, pp. 843-850, 1996.

[34] E. Asahina. Frost injury in living cells. Nature. Vol. 196, pp. 445-446, 1962.

[35] P.L. Steponkus, D. Stout, J. Wolfe, R. Lovelace. Freeze-induced electrical transients and cryoinjury, Cryo-Letters. Vol. 5, pp. 343-348, 1984.

[36] K. Muldrew and L.E. McGann. Mechanisms of intracellular ice formation, Biophys. J. Vol. 57, pp. 525532, 1990.

[37] M. Toner, E.G. Cravalho, M. Karel. Thermodynamics and kinetics of intracellular ice formation during freezing of biological cells, J. Appl. Phys. Vol. 67, pp. 1582-1593, 1990.

[38] J.O.M. Karlsson. A theoretical model of intracellular devitrification, Cryobiology. Vol. 42, pp. 154-169, 2001.

[39] J. Farrant and G.J. Morris. Thermal shock and dilution shock as the causes of freezing injury, Cryobiology. Vol. 10, pp. 134-140, 1973.

[40] J. Farrant, C.A. Walter, H. Lee, L.E. McGann. Use of two-step cooling procedures to examine factors influencing cell survival following freezing and thawing, Cryobiology. Vol. 14, pp. 273-286, 1977.

[41] J. Levitt. A sulfhydryl-disulfide hypothesis of frost injury and resistance in plants, J. Theor. Biol. Vol. 3, pp. 355-391,1962.

[42] M.J. Ashwood-Smith, G.W Morris, R. Fowler, T.C. Appleton, R. Ashorn. Physical factors are involved in the destruction of embryos and oocytes during freezing and thawing procedures, Hum. Reprod. Vol. 3, pp.795$802,1988$.

[43] G.J. Morris and J.J. McGrath. Intracellular ice nucleation and gas bubble formation in spirogyra, CryoLetters. Vol. 2, pp. 341-352, 1981.

[44] P.L. Steponkus and M.F Dowgert. Gas bubble formation during intracellular ice formation. CryoLetters. Vol. 2, pp. 42-47, 1981.

[45] K. Shimada and E. Asahina. Visualization of intracellular ice crystals formed in very rapidly frozen cells at $-27^{\circ} \mathrm{C}$. Cryobiology. Vol. 12, pp. 209-218, 1975.

[46] J.C. Bischof and B. Rubinsky. Large ice crystals in the nucleus of rapidly frozen liver cells. Cryobiology. Vol. 30, pp. 597-603, 1993.

[47] T.H. Jang, S.C. Park, J.H. Yang, et al. Cryopreservation and its clinical applications. Integr Med Res. Vol. 6, No. 1, pp.12-18, 2017.

[48] H. Sieme, H. Oldenhof, W.F. Wolkers. Mode of action of cryoprotectants for sperm preservation. Anim Reprod Sci. Vol.169, pp.2-5, 2016.

[49] V. Berejnov, N. S. Husseini, O. A. Alsaied, R.E.
Thorne. Effects of cryoprotectant concentration and cooling rate on vitrification of aqueous solutions. J. Appl. Cryst. Vol. 39, pp. 244-251, 2006.

[50] J. Sztein, K. Noble, J. Farley, L. Mobraaten. Comparison of Permeating and Nonpermeating Cryoprotectants for Mouse Sperm Cryopreservation. Cryobiology. Vol. 42, No. 1, pp.28-39, 2001.

[51] G. D. Elliott, S. Wang, B.J. Fuller. Cryoprotectants: A review of the actions and applications of cryoprotective solutes that modulate cell recovery from ultra-low temperatures. Cryobiology. Vol. 76, pp.74- 91, 2017.

[52] B. P. Best. Cryoprotectant Toxicity: Facts, Issues, and Questions. Rejuvenation research, 18(5), 422-436, 2015.

[53] S. Tsai, G. Chong, P. J. Meng, C. Lin. Sugars as supplemental cryoprotectants for marine organisms. Rev Aquacult. Vol. 10, pp. 703-715, 2018.

[54] J. Buitink, M.M.A.E Claessens, M.A. Hemminga, F.A Hoekstra. Influence of water content and temperature on molecular mobility and intracellular glasses in seeds and pollen, Plant Physiol. Vol. 118, pp. 531-541, 1998.

[55] J.H. Crowe, J.F. Carpenter, L.M. Crowe. The role of vitrification in anhydrobiosis, Annu. Rev. Physiol. Vol. 60, pp. 73-103, 1998.

[56] J. Wolfe, G. Bryant. Freezing, drying, and/or vitrification of membrane-solute-water systems, Cryobiology. Vol. 39, pp. 103-129, 1999.

[57] B.P. Gaber, I. Chandrasekhar, N. Pattabiraman. The interaction of trehalose with the phospholipid bilayer: A molecular modeling study, in Membranes, Metabolism and Dry Organisms, Leopold, A.C., Ed., Cornell University Press, Ithaca, NY, pp. 231-241, 1986.

[58] J.H. Crowe, J.F. Carpenter, L.M. Crowe. Preserving dry biomaterials: The water replacement hypothesis, Part 1, Biopharm. Vol. 4, pp. 28-33, 1993.

[59] J.H. Crowe, J.F. Carpenter, L.M. Crowe. Preserving dry biomaterials: The water replacement hypothesis, Part 2, Biopharm. Vol. 5, pp. 40-43, 1993.

[60] J. M. Zhang, Y. Sheng, Y. Z Cao, H. Y Wang, Z. J Chen. Effects of cooling rates and ice-seeding temperatures on the cryopreservation of whole ovaries. Journal of assisted reproduction and genetics. Vol. 28, No.7, pp. 627-633, 2011.

[61] C.J. Hunt. Cryopreservation: Vitrification and Controlled Rate Cooling. Methods Mol Biol. Vol. 1590, pp.41-77, 2017.

[62] S. Sukumar, S.P Kar. Numerical analysis of an enhanced cooling rate cryopreservation process in a biological tissue, Journal of Thermal Biology. Vol. 81, pp. 146-153, 2019.

[63] D. Gao, J. K Critser. Mechanisms of Cryoinjury in Living Cells. ILAR Journal. Vol. 41, No. 4, pp.187-196, 2000 .

[64] S.P. Leibo, J. Farrant, P. Mazur, M.G. Hanna, L.H Smith. Effects of freezing of marrow stem cell suspensions: interaction of cooling and warming rates in the presence of PVP, sucrose and Glycerol. 
Cryobiology. Vol. 6, pp.315-332, 1979.

[65] D.G Whittingham. Some factors affecting embryo storage in laboratory animals, in: K. Elliot, J. Whelan (Eds.), The Freezing of Mammalian Embryos. Ciba Foundation 52, Elsevier, Amsterdam, pp. 97-127, 1977.

[66] G. Missous, B. Thammavongs, V.M. Dieuleveux, M. Guéguen, J.M Panoff. Improvement of the cryopreservation of the fungal starter Geotrichum candidum by artificial nucleation and temperature downshift control, Cryobiology. Vol. 55; pp. 66-71, 2007.

[67] Y. Sugawara, A. Ichi-Ishi. Application of "CryoSeeds" in the cryopreservation of cultured plant cells and tissues, Plant Tissue Cult. Lett. Vol. 9, pp. 4750, 1992.

[68] T. Kojima, T. Soma, N. Oguri. Effect of ice nucleation by droplet of immobilized silver iodide on freezing of rabbit and bovine embryos, Theriogeneology. Vol. 30; pp. 1199-1207, 1988.

[69] A. Petersen, H. Schneider, G. Rau, B. Glasmacher. A new approach for freezing of aqueous solutions under active control of the nucleation temperature, Cryobiology. Vol. 53; pp. 248-257, 2006.

[70] P.V Hobbs. Ice Physics, Clarendon Press, Oxford, 1974.

[71] S. Passot, I.C. Trelea, M. Marin, M. Galen, G.J. Morris, F. Fonseca. Effects of controlled ice nuicleation on primary drying stage and protein receovery in vials cooled in a modified freeze-dryer, J. Biomech. Eng.
Vol. 131; pp. 1-5, 2009.

[72] R. Heidemann, M. Mered, D.Q. Wang, B. Gardner, C. Zhang, J. Michaels, H. J. Henzler, N. Abbasa, K. Konstantinov. A new seed train expansion method for recombinant mammalian cell lines, Cytotechnology. Vol 38; pp 99-108, 2002.

[73] A.K. Konstantinidis, W. Kuu, L. Otten, S.L. Nail, R.R. Sever. Controlled nucleation in freeze drying: effects of pore size in the dried product layer, mass transfer resistance, and primary drying rate, J. Pharm. Sci. Vol 100, pp. 3453-3470, 2011.

[74] G.M. Fahy. The relevance of cryoprotectant "toxicity" to cryobiology. Cryobiology. Vol. 23, No. 1, pp.1-13, 1986.

[75] G. Verheyen, I. Pletincx, A. Van Steirteghem. Effect of freezing method, thawing temperature and post-thaw dilution/washing on motility (CASA) and morphology characteristics of high-quality human sperm. Hum Reprod. Vol. 8, No.10, pp.1678-84, 1993.

[76] J.O. Nöthling, R. Shuttleworth. The effect of straw size, freezing rate and thawing rate upon post-thaw quality of dog semen. Theriogenology. Vol. 15, No.5, pp.1469-80, 2005.

[77] Y. Sharma, M. Sharma. Sperm cryopreservation: Principles and Biology. J Infertil Reprod Biol. Vol. 8, No. 3, pp. 43- 48, 2020.

[78] M. Warkentin, V. Berejnov, R.E. Thorne. Hyperquenching for Protein Crystallography. J. Appl. Cryst. Vol. 39, pp. 805-811, 2006. 